\title{
Surface Wave Propagation in a Generalized Thermoelastic Material with Voids
}

\author{
Baljeet Singh $^{1}$, Raj Pal ${ }^{2}$ \\ ${ }^{1}$ Department of Mathematics, Post Graduate Government College, Chandigarh, India \\ ${ }^{2}$ Department of Mathematics, Government Post Graduate College, Hisar, India \\ E-mail: bsinghgc11@gmail.com \\ Received February 21, 2011; revised March 6, 2011; accepted March 10, 2011
}

\begin{abstract}
In the present paper, the propagation of surface wave in a generalized thermoelastic solid with voids is considered. The governing equations are solved to obtain the general solution in $x-z$ plane. The appropriate boundary conditions at an interface between two dissimilar half-spaces are satisfied by appropriate particular solutions to obtain the frequency equation of the surface wave in the medium. Some special cases are also discussed.
\end{abstract}

Keywords: Thermoelasticity, Surface Waves, Boundary Conditions, Voids

\section{Introduction}

Theory of linear elastic materials with voids is an important generalization of the classical theory of elasticity. The theory is used for investigating various types of geological and biological materials for which classical theory of elasticity is not adequate. The theory of linear elastic materials with voids deals the materials with a distribution of small pores or voids, where the volume of void is included among the kinematics variables. The theory reduces to the classical theory in the limiting case of volume of void tending to zero. Non-linear theory of elastic materials with voids was developed by Nunziato and Cowin [1]. Cowin and Nunziato [2] developed a theory of linear elastic materials with voids to study mathematically the mechanical behavior of porous solids. Puri and Cowin [3] studied the behavior of plane waves in a linear elastic material with voids. Iesan [4] developed the linear theory of thermoelastic materials with voids.

Dhaliwal and Wang [5] formulated the heat-flux dependent thermoelasticity theory for an elastic material with voids. This theory includes the heat-flux among the constitutive variables and assumes an evolution equation for the heat-flux. Ciarletta and Scalia [6] developed a nonlinear theory of non-simple thermoelastic materials with voids. Ciarletta and Scarpetta [7] studied some results on thermoelasticity for dielectric materials with voids. Marin [8-9] studied uniqueness and domain of influence results in thermoelastic bodies with voids. Chirita and Scalia [10] studied the spatial and temporal behavior in linear thermoelasticity of materials with voids. A theory of thermoelastic materials with voids and without energy dissipation is developed by Cicco and Diaco [11]. Ciarletta et al. [12] presented a model for acoustic wave propagation in a porous material which also allows for propagation of a thermal displacement wave. Singh [13] studied the wave propagation in a homogeneous, isotropic generalized thermoelastic half space with voids in context of Lord and Shulman theory. Ciarletta et al. [14] studied the linear theory of micropolar thermoelasticity for materials with voids. Recently, Aoudai [15] derived the equations of the linear theory of thermoelastic diffusion in porous media based on the concept of volume fraction.

Lord Rayleigh [16] investigated the surface wave along the plane surface of an elastic solid. Chandrasekharaiah [17] discussed the effect of voids on Rayleigh waves in an elastic solid with voids and on Rayleigh-lamb waves in homogeneous elastic plate with voids. Many researchers have studied the surface waves in various theories of thermoelasticity. For example, Chadwick and Windle [18], Agarwal [19], Sharma and Singh [20], Mayer [21], Semerak [22], Chandrasekharaiah [23], Sharma et al. [24], Sharma and Kaur [25] and many others.

The present paper is motivated by the linear theory of thermoelasticity with voids developed by Iesan [4]. In Section 2, the governing equations are generalized with the help of Lord and Shulman [26] theory. In Section 3, 
these equations are solved for general solutions. In Sections 4 and 5 , the particular solutions are obtained and applied at required boundary conditions to obtain the frequency equation of surface waves in thermoelastic material with voids. In Section 6, some limiting cases of the problem are discussed. In last section, some concluding remarks are given.

\section{Governing Equations}

Following, Iesan [4] and Lord and Shulman [26], the constitutive equations and field equations in terms of the displacement, volume fraction and temperature, for homogeneous isotropic generalized thermo-elastic material with voids in the absence of the body forces, heat sources and extrinsic equilibrated body forces are

$$
\begin{gathered}
\sigma_{i j}=2 \mu e_{i j}+\delta_{i j}\left[\lambda e_{k k}-\beta \Theta+b \Phi\right] \\
q_{i}+\tau_{0} \ddot{q}_{i}=K \Theta_{, i} \\
h_{i}=\alpha \Phi_{, i} \\
\rho \eta=\beta e_{k k}+a \Theta+m \Phi \\
g=-b e_{k k}-\xi \Phi+m \Theta \\
\rho T_{0} \dot{\eta}=q_{i, i} \\
\mu u_{i, j j}+(\lambda+\mu) u_{j, i j}-\beta \Theta_{, i}+b \Phi_{, i}=\rho \ddot{u}_{i} \\
\rho c_{E}\left(\dot{\Theta}+\tau_{0} \ddot{\Theta}\right)+\beta T_{0}\left(\dot{u}_{k, k}+\tau_{0} \ddot{u}_{k, k}\right) \\
+m T_{0}\left(\dot{\Phi}+\tau_{0} \ddot{\Phi}\right)=K \Theta_{, i i} \\
\alpha \Phi_{, i i}-b u_{k, k}-\xi \Phi+m \Theta=\rho \chi \ddot{\Phi}
\end{gathered}
$$

where $\lambda, \mu$ are Lame's constants. $\Theta=T-T_{0}, T_{0}$ is the temperature of the medium in its natural state assumed to be such that $\left|\Theta / T_{0}\right| \ll 1, T$ is the absolute temperature, $\sigma_{i j}$ are the components of the stress tensor, $e_{i j}=\frac{1}{2}\left(u_{i, j}+u_{j, i}\right), u_{i}$ are the components of the displacement vector, $\eta$ is the entropy per unit mass, $K$ is the coefficient of thermal conductivity, $\tau_{0}$ is the thermal relaxation time. $\alpha, b, \xi$ are void material parameters, $\mathrm{m}$ is thermo-void coefficient, $\beta=(3 \lambda+2 \mu) \alpha_{t}, \alpha_{t}$ is the coefficient of linear thermal expansion, $\delta_{i j}$ is Kronecker delta, $q_{i}$ are the components of heat flux vector, $h_{i}$ are the components of equilibrated stress tensor, $\Phi$ is change in volume fraction field, $g$ is the intrinsic equilibrated body force and $a$ is thermal constant.

The Equations (7) to (9) are written in $x$-z plane as

$$
\begin{aligned}
& (\lambda+2 \mu) \frac{\partial^{2} u_{1}}{\partial x^{2}}+(\lambda+\mu) \frac{\partial^{2} u_{3}}{\partial x \partial z}+\mu \frac{\partial^{2} u_{1}}{\partial z^{2}} \\
& -\beta \frac{\partial \Theta}{\partial x}+b \frac{\partial \Phi}{\partial x}=\rho \frac{\partial^{2} u_{1}}{\partial t^{2}},
\end{aligned}
$$

$$
\begin{gathered}
(\lambda+2 \mu) \frac{\partial^{2} u_{3}}{\partial z^{2}}+(\lambda+\mu) \frac{\partial^{2} u_{1}}{\partial z \partial x}+\mu \frac{\partial^{2} u_{3}}{\partial x^{2}} \\
-\beta \frac{\partial \Theta}{\partial z}+b \frac{\partial \Phi}{\partial z}=\rho \frac{\partial^{2} u_{3}}{\partial t^{2}} \\
\rho c_{E} \tau \frac{\partial \Theta}{\partial t}+\beta T_{0} \tau\left(\frac{\partial^{2} u_{1}}{\partial x \partial t}+\frac{\partial^{2} u_{3}}{\partial z \partial t}\right)+m T_{0} \tau \frac{\partial \Phi}{\partial t} \\
=K\left(\frac{\partial^{2} \Theta}{\partial x^{2}}+\frac{\partial^{2} \Theta}{\partial z^{2}}\right), \\
\alpha\left(\frac{\partial^{2} \Phi}{\partial x^{2}}+\frac{\partial^{2} \Phi}{\partial z^{2}}\right)-b\left(\frac{\partial u_{1}}{\partial x}+\frac{\partial u_{3}}{\partial z}\right) \\
-\xi \Phi+m \Theta=\rho \chi \frac{\partial^{2} \Phi}{\partial t^{2}},
\end{gathered}
$$

where $\tau=1+\tau_{0} \frac{\partial}{\partial t}$.

Now the displacement components $u_{1}$ and $u_{3}$ are written in terms of potential function $\phi$ and $\psi$ as

$$
u_{1}=\frac{\partial \phi}{\partial x}-\frac{\partial \psi}{\partial z}, u_{3}=\frac{\partial \phi}{\partial z}+\frac{\partial \psi}{\partial x}
$$
have

Using Equation (14) into Equations (10) to (13), we

$$
\begin{gathered}
\mu\left(\frac{\partial^{2} \psi}{\partial x^{2}}+\frac{\partial^{2} \psi}{\partial z^{2}}\right)=\rho \frac{\partial^{2} \psi}{\partial t^{2}}, \\
(\lambda+2 \mu)\left(\frac{\partial^{2} \phi}{\partial x^{2}}+\frac{\partial^{2} \phi}{\partial z^{2}}\right)+b \Phi-\beta \Theta=\rho \frac{\partial^{2} \phi}{\partial t^{2}}, \\
\omega^{*} \nabla^{2} \Theta=\tau\left[\frac{\partial \Theta}{\partial t}+\varepsilon \frac{\partial}{\partial t}\left(\frac{\partial^{2} \phi}{\partial x^{2}}+\frac{\partial^{2} \phi}{\partial z^{2}}\right)+\varepsilon_{1} \frac{\partial \Phi}{\partial t}\right] \\
\alpha\left(\frac{\partial^{2} \Phi}{\partial x^{2}}+\frac{\partial^{2} \Phi}{\partial z^{2}}\right)-b\left(\frac{\partial^{2} \phi}{\partial x^{2}}+\frac{\partial^{2} \phi}{\partial z^{2}}\right)-\xi \Phi+m \Theta=\rho \chi \frac{\partial^{2} \Phi}{\partial t^{2}},
\end{gathered}
$$

where,

$$
\omega^{*}=\frac{K}{\rho c_{E}}, \varepsilon=\frac{\beta T_{0}}{\rho c_{E}}, \varepsilon_{1}=\frac{m T_{0}}{\rho c_{E}} .
$$

Here Equation (15) is uncoupled, whereas Equations (16), (17) and (18) are coupled in $\phi, \Theta$ and $\Phi$.

\section{Solutions}

To solve the Equations (16) to (18), we consider

$$
(\phi, \Theta, \Phi)=\{\bar{\phi}(z), \bar{\Theta}(z), \bar{\Phi}(z)\} \mathrm{e}^{i k(x-c t)},
$$

Substituting (19) in Equations (16)-(18), we obtain 
$\left[D^{2}+k^{2}\left(\Omega^{2}-1\right)\right] \bar{\phi}(z)-\beta_{1} \bar{\Theta}(z)+b_{1} \bar{\Phi}(z)=0$,

$$
\begin{aligned}
& i \varepsilon \omega \tau\left(-k^{2}+D^{2}\right) \bar{\phi}(z)+\left[\omega^{*}\left(-k^{2}+D^{2}\right)+i \tau \omega\right] \bar{\Theta}(z) \\
& +i \varepsilon_{1} \omega \tau \bar{\Phi}(z)=0
\end{aligned}
$$

$$
\begin{aligned}
& -b\left(-k^{2}+D^{2}\right) \bar{\phi}(z)+m \bar{\Theta}(z) \\
& +\left[\alpha\left(-k^{2}+D^{2}\right)-\xi+\rho \chi \omega^{2}\right] \bar{\Phi}(z)=0
\end{aligned}
$$

where,

$$
\begin{aligned}
& D^{2}=\frac{\mathrm{d}^{2}}{\mathrm{~d} z^{2}}, \Omega^{2}=\frac{\rho c^{2}}{\lambda+2 \mu}, \beta_{1}=\frac{\beta}{\lambda+2 \mu} \text { and } \\
& b_{1}=\frac{b}{\lambda+2 \mu}, \omega=k c
\end{aligned}
$$

The non-trivial solutions of Equations (20) to (22) exist if

$$
L_{0} D^{6}+L_{1} D^{4}+L_{2} D^{2}+L_{3}=0
$$

where

$$
\begin{aligned}
L_{0}= & \omega^{*} \alpha \\
L_{1}= & \alpha \omega^{*} k^{2}\left(\Omega^{2}-1\right)+i \beta_{1} \alpha \varepsilon \tau \omega-b b_{1} \omega^{2}-2 \omega^{*} \alpha k^{2} \\
& -\omega^{*} \xi+\omega^{*} \rho \omega \chi+i \omega \alpha \\
L_{2}= & k^{2}\left(\Omega^{2}-1\right)\left(-2 \omega^{*} \alpha k^{2}-\omega^{*} \xi+\omega^{*} \rho \omega \chi+i \omega \alpha\right) \\
& +\omega^{*} \alpha k^{4}+\omega^{*} b^{2} \xi-\omega^{*} k^{2} \rho \chi \omega^{2}-i \omega \tau k^{2} \alpha-i \omega \tau \xi \\
& +i \omega^{3} \rho \tau \chi+i m \xi \tau \omega-2 \alpha \beta_{1} \varepsilon \tau \omega k^{2} \\
& -i \beta_{1} \varepsilon \tau \omega \xi+i \beta_{1} \xi \tau \omega^{3} \rho \chi+i \beta_{1} \xi \tau \omega b+i b_{1} \varepsilon m \tau \omega \\
& +b b_{1} \omega^{2} k^{2}+b b_{1} \omega^{*} k^{2}-i b_{1} \omega b \tau \\
L_{3}= & k^{2}\left(\Omega^{2}-1\right)\left(\omega^{*} \alpha k^{4}+\omega^{*} b^{2} \xi-\omega^{*} b^{2} \rho \chi \omega^{2}\right. \\
& \left.-i \omega \tau k^{2} \alpha-i \omega \tau \xi+i \omega^{3} \rho \tau \chi-i m \xi \tau \omega\right)+i \beta_{1} \alpha \varepsilon \tau \omega k^{4} \\
& +i \beta_{1} \tau \xi \omega k^{2}-i \beta_{1} \varepsilon \tau \omega^{3} \rho k^{2} \chi-i \beta_{1} \xi \rho \omega b k^{2} \\
& -i b_{1} m \varepsilon \tau \omega k^{2}+b^{2} b_{1} k^{4} \omega^{*}+i b b_{1} \omega \tau k^{2}
\end{aligned}
$$

Let $m_{1}, m_{2}, m_{3}$ be the roots of the auxiliary Equation (23), then the general solutions $\phi, \Theta$ and $\Phi$ are written as

$$
\begin{gathered}
\phi=\left(A_{1} \mathrm{e}^{-m_{1} z}+A_{2} \mathrm{e}^{-m_{2} z}+A_{3} \mathrm{e}^{-m_{3} z}+A_{4} \mathrm{e}^{m_{1} z}\right. \\
\left.+A_{5} \mathrm{e}^{m_{2} z}+A_{6} \mathrm{e}^{m_{3} z}\right) \mathrm{e}^{i k(x-c t)} \\
\Theta=\left(\zeta_{1} A_{1} \mathrm{e}^{-m_{1} z}+\zeta_{2} A_{2} \mathrm{e}^{-m_{2} z}+\zeta_{3} A_{3} \mathrm{e}^{-m_{3} z}+\zeta_{1} A_{4} \mathrm{e}^{m_{1} z}\right. \\
\left.+\zeta_{2} A_{5} \mathrm{e}^{m_{2} z}+\zeta_{3} A_{6} \mathrm{e}^{m_{3} z}\right) \mathrm{e}^{i k(x-c t)} \\
\Phi=\left(\eta_{1} A_{1} \mathrm{e}^{-m_{1} z}+\eta_{2} A_{2} \mathrm{e}^{-m_{2} z}+\eta_{3} A_{3} \mathrm{e}^{-m_{3} z}+\eta_{1} A_{4} \mathrm{e}^{m_{1} z}\right. \\
\left.+\eta_{2} A_{5} \mathrm{e}^{m_{2} z}+\eta_{3} A_{6} \mathrm{e}^{m_{3} z}\right) \mathrm{e}^{i k(x-c t)}
\end{gathered}
$$

where

$$
\begin{gathered}
\zeta_{i}=\frac{\left(m_{i}^{2}-k^{2}\right)\left[b^{2}-G_{i}(\lambda+2 \mu)\right]+G_{i} \rho \omega^{2}}{\rho G_{i}+m b}, i=(1,2,3) \\
\eta_{i}=\frac{1}{b}\left[\left(k^{2}-m_{i}^{2}\right)(\lambda+2 \mu)-\rho \omega^{2}\right]+\frac{\beta}{b} \zeta_{i}, i=(1,2,3) \\
G_{i}=\chi \rho \omega^{2}-\alpha\left(k^{2}-m_{i}^{2}\right)-\xi
\end{gathered}
$$

The general solution $\psi$ of Equation (15) is written as

$$
\psi=\left(B_{0}^{*} \mathrm{e}^{-m_{4} z}+B_{1}^{*} \mathrm{e}^{m_{4} z}\right) \mathrm{e}^{i k(x-c t)},
$$

where, $m_{4}^{2}=k^{2}\left(1-\frac{\rho}{\mu} c^{2}\right)$.

\section{Formulation of the Problem}

Let us consider two semi-infinite half-spaces of thermoelastic solid with voids in welded contact as shown in Figure 1. The particular solutions in half-spaces $M$ and $M^{\prime}$ are as follows:

For medium $M$,

$$
\begin{gathered}
\phi=\left(A_{1} \mathrm{e}^{-m_{1} z}+A_{2} \mathrm{e}^{-m_{2} z}+A_{3} \mathrm{e}^{-m_{3} z}\right) \mathrm{e}^{i k(x-c t)} \\
\Theta=\left(\zeta_{1} A_{1} \mathrm{e}^{-m_{1} z}+\zeta_{2} A_{2} \mathrm{e}^{-m_{2} z}+\zeta_{3} A_{3} \mathrm{e}^{-m_{3} z}\right) \mathrm{e}^{i k(x-c t)} \\
\Phi=\left(\eta_{1} A_{1} \mathrm{e}^{-m_{1} z}+\eta_{2} A_{2} \mathrm{e}^{-m_{2} z}+\eta_{3} A_{3} \mathrm{e}^{-m_{3} z}\right) \mathrm{e}^{i k(x-c t)} \\
\psi=B_{1} \mathrm{e}^{-m_{4} z+i k(x-c t)}
\end{gathered}
$$

where,

$$
\begin{aligned}
& m_{1}{ }^{2}+m_{2}{ }^{2}+m_{3}{ }^{2}=-\frac{L_{1}}{L_{0}}, \\
& m_{1}{ }^{2} m_{2}{ }^{2}+m_{2}{ }^{2} m_{3}{ }^{2}+m_{3}{ }^{2} m_{1}{ }^{2}=\frac{L_{2}}{L_{0}}, m_{1}{ }^{2} m_{2}{ }^{2} m_{3}{ }^{2}=-\frac{L_{3}}{L_{0}}
\end{aligned}
$$

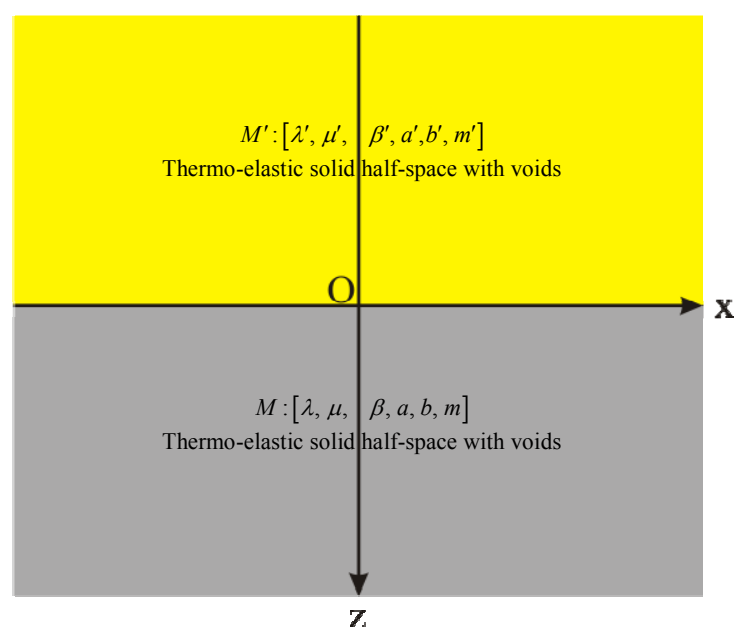

Figure 1. Geometry of the problem. 
Similarly, for medium $M^{\prime}$

$$
\begin{gathered}
\phi^{\prime}=\left(A_{1}^{\prime} \mathrm{e}^{m_{1}^{\prime} z}+A_{2}^{\prime} \mathrm{e}^{m_{2}^{\prime} z}+A_{3}^{\prime} \mathrm{e}^{m_{3}^{\prime} z}\right) \mathrm{e}^{i k(x-c t)} \\
\Theta^{\prime}=\left(\zeta_{1}^{\prime} A_{1}^{\prime} \mathrm{e}^{m_{1}^{\prime} z}+\zeta_{2}^{\prime} A_{2}^{\prime} \mathrm{e}^{m_{2}^{\prime} z}+\zeta_{3}^{\prime} A_{3}^{\prime} \mathrm{e}^{m_{3}^{\prime} z}\right) \mathrm{e}^{i k(x-c t)} \\
\Phi^{\prime}=\left(\eta_{1}^{\prime} A_{1}^{\prime} \mathrm{e}^{m_{1}^{\prime} z}+\eta_{2}^{\prime} A_{2}^{\prime} \mathrm{e}^{m_{2}^{\prime} z}+\eta_{3}^{\prime} A_{3}^{\prime} \mathrm{e}^{m_{3}^{\prime} z}\right) \mathrm{e}^{i k(x-c t)} \\
\psi^{\prime}=B_{1}^{\prime} \mathrm{e}^{m_{4}^{\prime} z+i k(x-c t)}
\end{gathered}
$$

Here, the symbols with primes in the following sections correspond to medium $M^{\prime}$.

\section{Boundary Conditions}

The boundary conditions at the interface $z=0$ are the continuity of force stress components, displacement components, heat flux, temperature and volume fractional field, i.e.

$$
\begin{aligned}
& \sigma_{z z}=\sigma_{z z}^{\prime}, \sigma_{z x}=\sigma_{z x}^{\prime}, \\
& \frac{\partial \Theta}{\partial z}=\chi_{1} \frac{\partial \Theta^{\prime}}{\partial z}, \frac{\partial \Phi}{\partial z}=\chi_{2} \frac{\partial \Phi^{\prime}}{\partial z}, \\
& u_{3}=u_{3}^{\prime}, u_{1}=u_{1}^{\prime}, \Theta=\Theta^{\prime}, \Phi=\Phi^{\prime} .
\end{aligned}
$$

where,

$$
\begin{aligned}
& \sigma_{z z}=2 \mu e_{z z}+\lambda e_{k k}-\beta \Theta+b \Phi, \\
& \sigma_{z z}^{\prime}=2 \mu^{\prime} e_{z z}^{\prime}+\lambda^{\prime} e_{k k}^{\prime}-\beta^{\prime} \Theta^{\prime}+b^{\prime} \Phi^{\prime} \\
& \sigma_{z x}=2 \mu e_{z x}, \sigma_{z x}^{\prime}=2 \mu^{\prime} e^{\prime}{ }_{z x}, \\
& u_{3}=\frac{\partial \phi}{\partial z}+\frac{\partial \psi}{\partial x}, u_{3}^{\prime}=\frac{\partial \phi^{\prime}}{\partial z}+\frac{\partial \psi^{\prime}}{\partial x} \\
& u_{1}=\frac{\partial \phi}{\partial x}-\frac{\partial \psi}{\partial z}, u_{1}^{\prime}=\frac{\partial \phi^{\prime}}{\partial x}-\frac{\partial \psi^{\prime}}{\partial z}, \\
& \chi_{1}=\frac{K^{\prime}}{K} \frac{1-i k c \tau_{0}}{1-i k c \tau_{0}^{\prime}}, \chi_{2}=\frac{\alpha^{\prime}}{\alpha}
\end{aligned}
$$

The particular solutions (29) to (35) satisfy the above boundary conditions if

$$
\left|\begin{array}{llllllll}
a_{11} & a_{12} & a_{13} & a_{14} & a_{15} & a_{16} & a_{17} & a_{18} \\
a_{21} & a_{22} & a_{23} & a_{24} & a_{25} & a_{26} & a_{27} & a_{28} \\
a_{31} & a_{32} & a_{33} & a_{34} & a_{35} & a_{36} & a_{37} & a_{38} \\
a_{41} & a_{42} & a_{43} & a_{44} & a_{45} & a_{46} & a_{47} & a_{48} \\
a_{51} & a_{52} & a_{53} & a_{54} & a_{55} & a_{56} & a_{57} & a_{58} \\
a_{61} & a_{62} & a_{63} & a_{64} & a_{65} & a_{66} & a_{67} & a_{68} \\
a_{71} & a_{72} & a_{73} & a_{74} & a_{75} & a_{76} & a_{77} & a_{78} \\
a_{81} & a_{82} & a_{83} & a_{84} & a_{85} & a_{86} & a_{87} & a_{88}
\end{array}\right|=0
$$

where

$$
\begin{aligned}
& a_{11}=-\lambda k^{2}+(\lambda+2 \mu) m_{1}^{2}-\beta \zeta_{1}+b \eta_{1} \\
& a_{12}=-\lambda k^{2}+(\lambda+2 \mu) m_{2}^{2}-\beta \zeta_{2}+b \eta_{2}
\end{aligned}
$$

$$
\begin{aligned}
& a_{13}=-\lambda k^{2}+(\lambda+2 \mu) m_{3}^{2}-\beta \zeta_{3}+b \eta_{3}, \\
& a_{14}=-2 i \mu \mathrm{km}_{4} \text {, } \\
& a_{15}=\lambda^{\prime} k^{2}-\left(\lambda^{\prime}+2 \mu^{\prime}\right) m_{1}^{\prime 2}+\beta^{\prime} \zeta_{1}^{\prime}-b^{\prime} \eta_{1}^{\prime}, \\
& a_{16}=\lambda^{\prime} k^{2}-\left(\lambda^{\prime}+2 \mu^{\prime}\right) m_{2}^{\prime 2}+\beta^{\prime} \zeta_{2}^{\prime}-b^{\prime} \eta_{2}^{\prime} \text {, } \\
& a_{17}=\lambda^{\prime} k^{2}-\left(\lambda^{\prime}+2 \mu^{\prime}\right) m_{3}^{\prime 2}+\beta^{\prime} \zeta_{3}^{\prime}-b^{\prime} \eta_{3}^{\prime} \text {, } \\
& a_{18}=-2 i \mu^{\prime} k m_{4}^{\prime}, a_{21}=-2 i \mu k m_{1} \text {, } \\
& a_{22}=-2 i \mu k m_{2}, a_{23}=-2 i \mu k m_{3} \text {, } \\
& a_{24}=-\mu\left(k^{2}+m_{4}^{2}\right), a_{25}=-2 i \mu^{\prime} k m_{1}^{\prime}, a_{26}=-2 i \mu^{\prime} k m_{2}^{\prime} \text {, } \\
& a_{27}=-2 i \mu^{\prime} k m_{3}^{\prime}, a_{28}=\mu^{\prime}\left(k^{2}+m_{4}^{\prime 2}\right) \text {. } \\
& a_{31}=m_{1} \zeta_{1}, a_{32}=m_{2} \zeta_{2}, a_{33}=m_{3} \zeta_{3}, a_{34}=0 \text {, } \\
& a_{35}=m_{1}^{\prime} \chi_{1} \zeta_{1}^{\prime}, a_{36}=m_{2}^{\prime} \chi_{1} \zeta_{2}^{\prime}, a_{37}=m_{3}^{\prime} \chi_{1} \zeta_{3}^{\prime} \text {, } \\
& a_{38}=0 \text {. } \\
& a_{41}=m_{1} \eta_{1}, a_{42}=m_{2} \eta_{2}, a_{43}=m_{3} \eta_{3}, a_{44}=0 \text {, } \\
& a_{45}=m_{1}^{\prime} \eta_{1}^{\prime} \chi_{2}, a_{46}=m_{2}^{\prime} \eta_{2}^{\prime} \chi_{2}, a_{47}=m_{3}^{\prime} \eta_{3}^{\prime} \chi_{2} \text {, } \\
& a_{48}=0 \text {. } \\
& a_{51}=m_{1}, a_{52}=m_{2}, a_{53}=m_{3}, a_{54}=-i k, \\
& a_{55}=m_{1}^{\prime}, a_{56}=m_{2}^{\prime}, a_{57}=m_{3}^{\prime}, a_{58}=i k \text {. } \\
& a_{61}=i k, a_{62}=i k, a_{63}=i k, a_{64}=m_{4} \text {, } \\
& a_{65}=-i k, a_{66}=-i k, a_{67}=-i k, a_{68}=m_{4}^{\prime} \text {. } \\
& a_{71}=\zeta_{1}, a_{72}=\zeta_{2}, a_{73}=\zeta_{3}, a_{74}=0 \text {, } \\
& a_{75}=-\zeta_{1}^{\prime}, a_{76}=-\zeta_{2}^{\prime}, a_{77}=-\zeta_{3}^{\prime}, a_{78}=0 \text {. } \\
& a_{81}=\eta_{1}, a_{82}=\eta_{2}, a_{83}=\eta_{3}, a_{84}=0 \text {, } \\
& a_{85}=-\eta_{1}^{\prime}, a_{86}=-\eta_{2}^{\prime}, a_{87}=-\eta_{3}^{\prime}, a_{88}=0 \text {. }
\end{aligned}
$$

The Equation (37) gives the frequency equation for surface wave in a generalized thermo-elastic medium with voids.

\section{Limiting Cases}

1) If we neglect the void parameters, then the Equation (37) reduces to

$$
\left|\begin{array}{llllll}
a_{11}^{\prime} & a_{12}^{\prime} & a_{13}^{\prime} & a_{14}^{\prime} & a_{15}^{\prime} & a_{16}^{\prime} \\
a_{21}^{\prime} & a_{22}^{\prime} & a_{23}^{\prime} & a_{24}^{\prime} & a_{25}^{\prime} & a_{26}^{\prime} \\
a_{31}^{\prime} & a_{32}^{\prime} & a_{33}^{\prime} & a_{34}^{\prime} & a_{35}^{\prime} & a_{36}^{\prime} \\
a_{41}^{\prime} & a_{42}^{\prime} & a_{43}^{\prime} & a_{44}^{\prime} & a_{45}^{\prime} & a_{46}^{\prime} \\
a_{51}^{\prime} & a_{52}^{\prime} & a_{53}^{\prime} & a_{54}^{\prime} & a_{55}^{\prime} & a_{56}^{\prime} \\
a_{61}^{\prime} & a_{62}^{\prime} & a_{63}^{\prime} & a_{64}^{\prime} & a_{65}^{\prime} & a_{66}^{\prime}
\end{array}\right|
$$

where,

$$
\begin{aligned}
& a_{11}^{\prime}=-\lambda k^{2}+(\lambda+2 \mu) m_{1}^{2}-\beta \zeta_{1}, \\
& a_{12}^{\prime}=-\lambda k^{2}+(\lambda+2 \mu) m_{2}^{2}-\beta \zeta_{2}, a_{13}^{\prime}=-2 i \mu k m_{4}, \\
& a_{14}^{\prime}=\lambda^{\prime} k^{2}-\left(\lambda^{\prime}+2 \mu^{\prime}\right) m_{1}^{\prime 2}+\beta^{\prime} \zeta_{1}^{\prime},
\end{aligned}
$$




$$
\begin{aligned}
& a_{15}^{\prime}=\lambda^{\prime} k^{2}-\left(\lambda^{\prime}+2 \mu^{\prime}\right) m_{2}^{\prime 2}+\beta^{\prime} \zeta_{2}^{\prime}, a_{16}^{\prime}=-2 i \mu^{\prime} k m_{4}^{\prime}, \\
& a_{21}^{\prime}=-2 i m_{1} \mu k, a_{22}^{\prime}=-2 i m_{2} \mu k, a_{23}^{\prime}=-\mu\left(k^{2}+m_{4}^{2}\right), \\
& a_{24}^{\prime}=-2 i m_{1}^{\prime} \mu^{\prime} k, a_{25}^{\prime}=-2 i m_{2}^{\prime} \mu^{\prime} k, a_{26}^{\prime}=\mu^{\prime}\left(k^{2}+m_{4}^{\prime 2}\right) \\
& a_{31}^{\prime}=m_{1} \zeta_{1}, a_{32}^{\prime}=m_{2} \zeta_{2}, a_{33}^{\prime}=0 \\
& a_{34}^{\prime}=\chi_{1} m_{1}^{\prime} \zeta_{1}^{\prime}, a_{35}^{\prime}=\chi_{1} m_{2}^{\prime} \zeta_{2}^{\prime}, a_{36}^{\prime}=0 \\
& a_{41}^{\prime}=m_{1}, a_{42}^{\prime}=m_{2}, a_{43}^{\prime}=-i k \\
& a_{44}^{\prime}=m_{1}^{\prime}, a_{45}^{\prime}=m_{2}^{\prime}, a_{46}^{\prime}=i k . \\
& a_{51}^{\prime}=i k, a_{52}^{\prime}=i k, a_{53}^{\prime}=m_{4}, \\
& a_{54}^{\prime}=-i k, a_{55}^{\prime}=-i k, a_{56}^{\prime}=m_{4}^{\prime} \\
& a_{61}^{\prime}=\zeta_{1}, a_{62}^{\prime}=\zeta_{2}, a_{63}^{\prime}=0 \\
& a_{64}^{\prime}=-\zeta_{1}^{\prime}, a_{65}^{\prime}=-\zeta_{2}^{\prime}, a_{66}^{\prime}=0 .
\end{aligned}
$$

The Equation (38) gives the frequency equation for surface waves in a generalized thermo-elastic medium.

2) If we neglect thermal parameters, then the Equation (37) reduces to

$$
\left|\begin{array}{llllll}
b_{11} & b_{12} & b_{13} & b_{14} & b_{15} & b_{16} \\
b_{21} & b_{22} & b_{23} & b_{24} & b_{25} & b_{26} \\
b_{31} & b_{32} & b_{33} & b_{34} & b_{35} & b_{36} \\
b_{41} & b_{42} & b_{43} & b_{44} & b_{45} & b_{46} \\
b_{51} & b_{52} & b_{53} & b_{54} & b_{55} & b_{56} \\
b_{61} & b_{62} & b_{63} & b_{64} & b_{65} & b_{66}
\end{array}\right|=0
$$

where

$$
\begin{aligned}
& b_{11}=-\lambda k^{2}+(\lambda+2 \mu) m_{1}^{2}+b \eta_{1}, \\
& b_{12}=-\lambda k^{2}+(\lambda+2 \mu) m_{3}^{2}+b \eta_{3}, b_{13}=-2 i \mu k m_{4}, \\
& b_{14}=\lambda^{\prime} k^{2}-\left(\lambda^{\prime}+2 \mu^{\prime}\right) m_{1}^{\prime 2}-b^{\prime} \eta_{1}^{\prime}, \\
& b_{15}=\lambda^{\prime} k^{2}-\left(\lambda^{\prime}+2 \mu^{\prime}\right) m_{3}^{\prime 2}-b^{\prime} \eta_{3}^{\prime}, b_{16}=-2 i \mu^{\prime} k m_{4}^{\prime}, \\
& b_{21}=-2 i m_{1} \mu k, b_{22}=-2 i m_{3} \mu k, b_{23}=-\mu\left(k^{2}+m_{4}^{2}\right), \\
& b_{24}=-2 i m_{1}^{\prime} \mu^{\prime} k, b_{25}=-2 i m_{3}^{\prime} \mu^{\prime} k, b_{26}=\mu^{\prime}\left(k^{2}+m_{4}^{\prime 2}\right) . \\
& b_{31}=m_{1} \eta_{1}, b_{32}=m_{3} \eta_{3}, b_{33}=0, \\
& b_{34}=\chi_{2} m_{1}^{\prime} \eta_{1}^{\prime}, b_{35}=\chi_{2} m_{3}^{\prime} \eta_{3}^{\prime}, b_{36}=0 . \\
& b_{41}=m_{1}, b_{42}=m_{3}, b_{43}=-i k, \\
& b_{44}=m_{1}^{\prime}, b_{45}=m_{3}^{\prime}, b_{46}=i k . \\
& b_{51}=i k, b_{52}=i k, b_{53}=m_{4}, \\
& b_{54}=-i k, b_{55}=-i k, b_{56}=m_{4}^{\prime} \\
& b_{61}=\eta_{1}, b_{62}=\eta_{3}, b_{63}=0, \\
& b_{64}=-\eta_{1}^{\prime}, b_{65}=-\eta_{3}^{\prime}, b_{66}=0 .
\end{aligned}
$$

The Equation (39) gives the frequency equation for surface wave in an elastic medium with voids.
3) If we neglect void and thermal parameters, then the Equation (37) reduces to

$$
\left|\begin{array}{llll}
c_{11} & c_{12} & c_{13} & c_{14} \\
c_{21} & c_{22} & c_{23} & c_{24} \\
c_{31} & c_{32} & c_{33} & c_{34} \\
c_{41} & c_{42} & c_{43} & c_{44}
\end{array}\right|=0
$$

where

$$
\begin{aligned}
& c_{11}=-\lambda k^{2}+(\lambda+2 \mu) m_{1}^{2}, c_{12}=-2 i \mu k m_{4}, \\
& c_{13}=\lambda^{\prime} k^{2}-\left(\lambda^{\prime}+2 \mu^{\prime}\right) m_{1}^{\prime 2}, c_{14}=2 i \mu^{\prime} k m_{4}^{\prime}, \\
& c_{21}=-2 i m_{1} \mu k, c_{22}=-\mu\left(k^{2}+m_{4}^{2}\right), \\
& c_{23}=-2 i m_{1}^{\prime} \mu^{\prime} k, c_{24}=\mu^{\prime}\left(k^{2}+m_{4}^{\prime 2}\right) . \\
& c_{31}=m_{1}, c_{32}=-i k, \\
& c_{33}=m_{1}^{\prime}, c_{34}=i k \\
& c_{41}=i k, c_{42}=m_{4}, \\
& c_{43}=-i k, c_{44}=m_{4}^{\prime} .
\end{aligned}
$$

The Equation (40) gives the frequency equation for surface wave in an elastic medium

4) If we remove the upper half-space, then the Equation (37) reduces to

$$
\left|\begin{array}{llll}
d_{11} & d_{12} & d_{13} & d_{14} \\
d_{21} & d_{22} & d_{23} & d_{24} \\
d_{31} & d_{32} & d_{33} & d_{34} \\
d_{41} & d_{42} & d_{43} & d_{44}
\end{array}\right|=0
$$

where

$$
\begin{aligned}
& d_{11}=-\lambda k^{2}+(\lambda+2 \mu) m_{1}^{2}-\beta \zeta_{1}+b \eta_{1}, \\
& d_{12}=-\lambda k^{2}+(\lambda+2 \mu) m_{2}^{2}-\beta \zeta_{2}+b \eta_{2}, \\
& d_{13}=-\lambda k^{2}+(\lambda+2 \mu) m_{3}^{2}-\beta \zeta_{3}+b \eta_{3}, \\
& d_{14}=-2 i \mu k m_{4} d_{21}=-2 i \mu k m_{1}, d_{22}=-2 i \mu k m_{2}, \\
& d_{23}=-2 i \mu k m_{3}, d_{24}=-\mu\left(k^{2}+m_{4}^{2}\right) . \\
& d_{31}=m_{1} \zeta_{1}, d_{32}=m_{2} \zeta_{2}, d_{33}=m_{3} \zeta_{3}, d_{34}=0 . \\
& d_{41}=m_{1} \eta_{1}, d_{42}=m_{2} \eta_{2}, d_{43}=m_{3} \eta_{3}, d_{44}=0 .
\end{aligned}
$$

The Equation (41) gives the frequency equation of a Rayleigh surface wave in a half-space of a generalized thermo-elastic material with void.

\section{Conclusions}

The frequency equation of surface waves in generalized thermoelastic material with voids is obtained. The frequency equation of Rayleigh surface wave is obtained as limiting case. The theoretical results indicate that the speed of surface wave depends on various material pa- 
rameters. Present analytical solutions can be used to find numerically the speed of surface wave for a particular material modeled as thermoelastic material with voids.

\section{References}

[1] J. W. Nunziato and S. C. Cowin, "A Nonlinear Theory of Elastic Materials with Voids," Archive for Rational Mechanics and Analysis, Vol. 72, No. 2, 1979, pp. 175-201.

[2] S. C. Cowin and J. W. Nunziato, "Linear Elastic Materials with Voids," Journal of Elasticity, Vol. 13, No. 2, 1983, 125-147. doi:10.1007/BF00041230

[3] P. Puri and S. C. Cowin, "Plane Waves in Linear Elastic Materials with Voids," Journal of Elasticity, Vol. 15, No. 2, 1985, pp. 167-183. doi:10.1007/BF00041991

[4] D. Iesan, "A Theory of Thermoelastic Materials with Voids," Acta Mechanica, Vol. 60, No. 1-2, 1986, pp. 6789. doi:10.1007/BF01302942

[5] R. S. Dhaliwal and J. Wang, "A Heat-Flux Dependent Theory of Thermoelasticity with Voids," Acta Mechanica, Vol. 110, No. 1-4, 1993, pp. 33-39.

[6] M. Ciarletta and A. Scalia, "On the Nonlinear Theory of Nonsimple Thermoelastic Materials with Voids," Journal of Applied Mathematics and Mechanics, Vol. 73, No. 2, 1993, pp. 67-75.

[7] M. Ciarletta and E. Scarpetta, "Some Results on TherMoelasticity for Dielectric Materials with Voids," Journal of Applied Mathematics and Mechanics, Vol. 75, No. 9, 1995, pp. 707-714.

[8] M. Marin, "A Uniqueness Result for Body with Voids in Linear Thermoelasticity," Rendiconti di Matematica, Vol. 17, No. 1, 1997, pp. 103-113.

[9] M. Marin, "On the Domain of Influence in Thermoelasticity of Bodies with Voids," Archiv der Mathematik, Vol. 33, No. 4, 1997, pp. 301-308.

[10] S. Chirita and A. Scalia, "On the Spatial and Temporal Behavior in Linear Thermoelasticity of Materials with Voids," Journal of Thermal Stresses, Vol. 24, No. 5, 2001, pp. 433-455.

[11] S. D. Cicco and M. Diaco, "A Theory of Thermoelastic Materials with Voids without Energy Dissipation," Journal of Thermal Stresses, Vol. 25, No. 5, 2002, pp. 493503. doi:10.1080/01495730252890203

[12] M. Ciarletta, B. Straughan and V. Zampoli, "Thermoporoacoustic Acceleration Waves in Elastic Materials with Voids without Energy Dissipation," International Journal of Engineering Science, Vol. 45, No. 9, 2007, pp. 736-743. doi:10.1016/j.ijengsci.2007.05.001

[13] B. Singh, "Wave Propagation in a Generalized Thermoelastic Material with Voids," Applied Mathematics and Computation, Vol. 189, No. 1, 2007, pp. 698-709. doi:10.1016/j.amc.2006.11.123
[14] M. Ciarletta, M. Svanadze and L. Buonanno, "Plane Waves and Vibrations in the Theory of Micropolar Thermoelasticity for Materials with Voids," European Journal of Mechanics-A-Solids, Vol. 28, No. 4, 2009, pp. 897-903. doi:10.1016/j.euromechsol.2009.03.008

[15] M. Aoudai, "A Theory of Thermoelastic Diffusion Material with Voids," Zeitschrift für Angewandte Mathematik und Physik, Vol. 61, No. 2, 2010, pp. 357-379. doi:10.1007/s00033-009-0016-0

[16] L. Rayleigh, "On Waves Propagating along the Plane Surface of an Elastic Solid," Proceedings of the London Mathematical Society, Vol. 17, No. 1, 1885, pp. 4-11. doi:10.1112/plms/s1-17.1.4

[17] D. S. Chandrasekharaiah, "Effects of Surface Stresses and Voids on Rayleigh Waves in an Elastic Solid," International Journal of Engineering Science, Vol. 25, No. 2, 1987, pp. 205-211.

[18] P. Chadwick and D. W. Windle, "Propagation of Rayleigh Waves along Isothermal and Insulated Boundaries," Proceedings of the Royal Society of America, Vol. 280, No. 1380, 1964, pp. 47-71. doi:10.1098/rspa.1964.0130

[19] V. K. Agarwal, "On Surface Waves in Generalized Thermoelasticity," Journal of Elasticity, Vol. 8, No. 2, 1978, pp. 171-177. doi:10.1007/BF00052480

[20] J. N. Sharma and H. Singh, "Thermoelastic Surface Waves in a Transversely Isotropic Half-Space with Thermal Relaxation," Indian Journal of Pure and Applied Mathematics, Vol. 16, No. 10, 1985, pp. 1202-1219.

[21] A. P. Mayer, "Thermoelastic Attenuation of Surface Acoustic Waves," International Journal of Engineering Science, Vol. 28, No. 10, 1990, pp. 1073-1082. doi:10.1016/0020-7225(90)90135-6

[22] F. V. Semerak, "The Effect of Thermal Relaxation on Rayleigh Surface Waves in a Thermoelastic Medium," Journal of Mathematical Sciences, Vol. 88, No. 3, 1997, pp. 396-399.

[23] D. S. Chandrasekharaiah, "Thermoelastic Rayleigh Waves without Energy Dissipation," Mechanics Research Communication, Vol. 24, No. 1, 1997, pp. 93-102. doi:10.1016/S0093-6413(96)00083-3

[24] J. N. Sharma, D. Singh and R. Kumar, "Generalized Thermoelastic Waves in Homogeneous Isotropic Plates," Journal of the Acoustical Society of America, Vol. 108, No. 2, 2000, pp. 848-851. doi:10.1121/1.429619

[25] J. N. Sharma and D. Kaur, "Rayleigh Waves in Rotating Thermoelastic Solids with Voids" International Journal of Applied Mathematics and Mechanics, Vol. 6, No. 3, 2010, pp. 43-61.

[26] H. W. Lord and Y. Shulman, "A Generalized Dynamical Theory of Thermoelasticity," Journal of the Mechanics and Physics of Solids, Vol. 15, No. 5, 1967, pp. 299-309. doi:10.1016/0022-5096(67)90024-5 